\title{
Mach Stem Height and Growth Rate Predictions
}

\author{
Christopher A. Mouton* and Hans G. Hornung \\ California Institute of Technology, Pasadena, California 91125 \\ DOI: $10.2514 / 1.27460$
}

\begin{abstract}
A new, more accurate prediction of Mach stem height in steady flow is presented. In addition, starting with a regular reflection in the dual-solution domain, the growth rate of the Mach stem from the time it is first formed till it reaches its steady-state height is presented. Comparisons between theory, experiments, and computations are presented for the Mach stem height. The theory for the Mach stem growth rate in both two and three dimensions is compared to computational results. The Mach stem growth theory provides an explanation for why, once formed, a Mach stem is relatively persistent.
\end{abstract}

\section{Nomenclature}

$\begin{array}{lll}g & = & \text { spacing between wedge and axis of symmetry } \\ M & = & \text { Mach number } \\ P & = & \text { pressure } \\ s & = & \text { Mach stem height } \\ U & = & \text { speed } \\ w & = & \text { wedge length } \\ \alpha & = & \text { leading shock angle with respect to the freestream } \\ \gamma & = & \text { ratio of specific heats } \\ \delta & = & \text { triple-point slip-line angle } \\ \theta & = & \text { wedge angle with respect to the freestream } \\ \mu & = & \text { Mach angle } \\ \rho & = & \text { density } \\ \phi & = & \text { triple-point reflected shock angle }\end{array}$

Subscripts

$a=$ conditions directly behind Mach stem

$\mathrm{Ms}=$ Mach stem properties

tp $=$ triple-point properties

$1=$ region 1 condition

$2=$ region 2 condition

$3=$ conditions between Mach stem and sonic throat

$\star \quad=\quad$ sonic throat condition

$\infty=$ freestream condition

Superscripts

tp $=$ triple-point reference frame

$+\quad=$ nondimensional quantity

\section{Introduction}

C ONSIDER the reflection of a shock, generated by a wedge in steady supersonic flow, from a wall (single wedge configuration) or from a plane of symmetry (double wedge configuration). For a sufficiently high freestream Mach number, there exists a range of wedge angles (the dual-solution domain) in which both regular and Mach reflections are possible. To date there is no accurate method of predicting the height of a Mach stem in steady flow. Predictions of Mach stem height can be important in the design of

Received 23 August 2006; revision received 6 March 2007; accepted for publication 6 March 2007. Copyright @ 2007 by the American Institute of Aeronautics and Astronautics, Inc. All rights reserved. Copies of this paper may be made for personal or internal use, on condition that the copier pay the $\$ 10.00$ per-copy fee to the Copyright Clearance Center, Inc., 222 Rosewood Drive, Danvers, MA 01923; include the code 0001-1452/07 \$10.00 in correspondence with the CCC.

${ }^{*}$ Graduate Student, Graduate Aeronautical Laboratories, 1200 East California Boulevard. AIAA Member.

Clarence L. Johnson Professor of Aeronautics, Director of GALCIT, Emeritus. Lifetime AIAA Fellow. supersonic inlets if the inlet is expected to experience Mach reflection. An accurate prediction of the Mach stem height may also be useful in understanding the behavior of the shock reflection in the dual-solution domain.

Azevedo [1, 2] (see also Ben-Dor [3] ) developed a theory based on the location of the sonic throat formed by the initially converging flow behind the Mach stem. However, his prediction consistently underestimated the actual Mach stem height. The primary drawback of his calculation is that the sonic throat of the converging flow formed behind the Mach stem is assumed to be at the point where the leading characteristic of the expansion fan intersects the slip stream. Also, Li and Ben-Dor [4] note that Azevedo uses a methodology, which overdefines the problem, and as a result there is no unique solution based on his formulation. The aims of the present work are to relax some of the assumptions made by Azevedo to obtain more accurate predictions of the Mach stem height and to analyze the rate of growth of a Mach stem starting from a regular reflection in the dual-solution domain. Work by Li and Ben-Dor [4] corrects some of the flaws in the theory of Azevedo, but gives very similar approximations of the Mach stem height, which differ significantly from the experimental work of Hornung and Robinson [5]. In particular, $\mathrm{Li}$ and Ben-Dor provide a methodology for solving for the Mach stem height that produces a unique solution. In addition, their work gives a Mach stem height of zero at the von Neumann condition. The primary difference in the results of Li and Ben-Dor is a shift in the Mach stem height curve, such that it intersects that von Neumann condition when the Mach stem height is zero, thus providing a slightly higher prediction for the Mach stem height. A brief comparison of the theoretical work by Azevedo, Li, and Ben-Dor and the current paper is presented in Table 1 . The works by Li et al. [6] and Schotz et al. [7] consider downstream influences on the Mach stem height; however, the theoretical work of Ben-Dor et al. [8] and the experimental work of Chpoun and Leclerc [9] show that the Mach stem height does not vary with downstream conditions. This is as expected, because the flow in the expansion region and downstream of the sonic throat is supersonic and therefore these influences cannot affect the Mach stem height. There is, therefore, no need in the current work to consider the flow downstream of the expansion wave corresponding to the sonic throat.

\section{Problem Setup}

The problem setup is shown graphically in Fig. 1 . We can either consider two opposing wedges, or for inviscid flow, a wedge above a flat plate. The wedge, with a length $w$, is declined at an angle $\theta_{1}$ with respect to the freestream flow and produces a shock at an angle $\alpha$. The height of the triple point above the surface is the Mach stem height, denoted $s$. In the case of two symmetric wedges, $s$ is half the total Mach stem height. At the triple point a slip line is created, which is initially declined at an angle $\delta$ with respect to the surface or plane of symmetry. The reflected shock from the triple point is inclined at an angle $\phi$ with respect to the surface. 
Table 1 Summary table comparing the methodology of the present paper to Azevedo [1,2], and Li and Ben-Dor [4]. $E F$ refers to the leading characteristic of the expansion fan originating from the aft corner of the wedge

\begin{tabular}{lccc}
\hline \hline & Azevedo [1, 2] $]$ & Li and Ben-Dor [4] & Present \\
\hline Sonic throat location & At $E F$ & Downstream of $E F$ & Downstream of $E F$ \\
Mach stem shape & Straight & Curved & Straight \\
Reflected wave through expansion & N/A & Curved & Kinked \\
Slip stream & Straight & Curved & Straight \\
Von Neumann condition & Not matched & Matched & Matched \\
Growing Mach stem & No & No & Yes \\
\hline \hline
\end{tabular}

In general the Mach stem height $s$ is a function of the Mach number $M$, the ratio of specific heats $\gamma$, the spacing between the wedge and the flat surface $g$, the angle of the wedge $\theta_{1}$, and the wedge length $w$. That is to say

$$
s^{+}=f^{+}\left(M, \gamma, g^{+}, \theta_{1}\right)
$$

where $f^{+}$is an unknown nondimensional function, $s^{+}=\frac{s}{w}$, and $g^{+}=\frac{g}{w}$. Normalizing lengths by $w$ is a good choice, because, in experiments, $w$ will almost always be a fixed length and not a function of the wedge angle $\theta_{1}$.

\section{Mass and Momentum Balance}

Azevedo [1,2] considers a problem setup as shown in Fig. 1 subject to several assumptions. First, he assumes that the sonic throat occurs where the leading characteristic of the expansion fan intersects the slip line. Second, he assumes that the region between the slip line and the symmetry plane, and between the Mach stem and the sonic throat is an isentropically converging ideal gas flow with a straight streamline $\overline{T H}$. To analyze the flow, Azevedo applies conservation of mass and momentum, and this derivation is included in Sec. I.

The result can be written in matrix form and solved; the matrix equation is

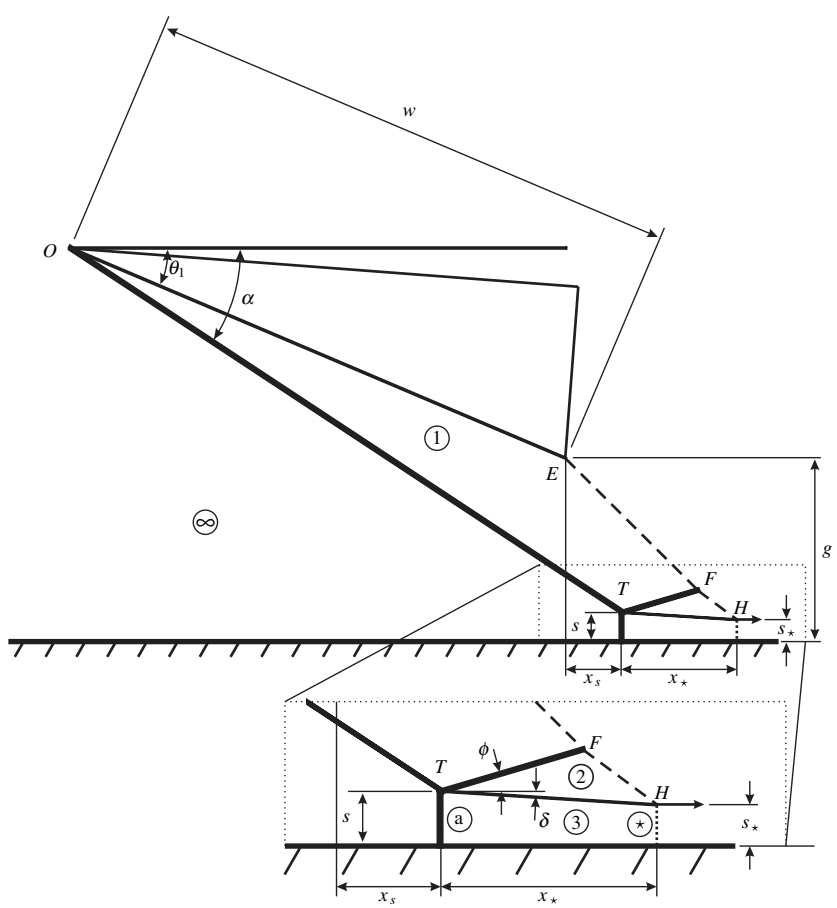

Fig. 1 Illustration of flow setup used by Azevedo [1,2] to predict the Mach stem height.

$$
\begin{gathered}
\left(\begin{array}{ccccc}
0 & 0 & A_{\mathrm{r}} & a_{14} & a_{15} \\
0 & 0 & a_{23} & a_{24} & a_{25} \\
\frac{1}{\gamma M_{\infty}^{2}} & P_{3}^{+} & 0 & a_{34} & a_{35} \\
1 & 1 & 0 & a_{44} & a_{45} \\
0 & 0 & 1 & a_{54} & a_{55}
\end{array}\right)\left(\begin{array}{c}
x_{s}^{+} \\
x_{\star}^{+} \\
s_{\star}^{+} \\
\overline{E F^{+}} \\
\overline{F H^{+}}
\end{array}\right) \\
=\left(\begin{array}{c}
g^{+}+\sin \theta_{1} \\
\frac{g^{+}+\sin \theta_{1}}{\gamma M_{\infty}^{2}}+g^{+}+\sin \theta_{1}-P_{1}^{+} \sin \theta_{1} \\
-\frac{1}{\gamma M_{\infty}^{2}} \cos \theta_{1}+P_{1}^{+} \cos \theta_{1} \\
0 \\
g^{+}
\end{array}\right.
\end{gathered}
$$

where

$$
\begin{gathered}
a_{14}=\frac{\sin \alpha}{\sin \left(\alpha-\theta_{1}\right)} \sin \mu_{1} \\
a_{15}=\frac{\sin \alpha \sin \left(\theta_{1}+\phi\right)}{\sin \left(\alpha-\theta_{1}\right) \sin (\phi+\delta)} \sin \mu_{2} \\
a_{24}=\gamma P_{1}^{+} M_{1} \cos \theta_{1}+P_{1}^{+} \sin \left(\mu_{1}+\theta_{1}\right) \\
a_{25}=\gamma P_{\star}^{+}+P_{\star}^{+} \\
a_{34}=\gamma P_{1}^{+} M_{2} \cos \delta+P_{2}^{+} \sin \left(\mu_{2}+\delta\right) \\
a_{55}=\sin \left(\mu_{2}+\delta\right) \\
a_{54}=-\sin \left(\mu_{1}+\theta_{1}^{+} \cos \left(\mu_{1}+\theta_{1}\right)\right. \\
a_{35}=\gamma P_{2}^{+} M_{2} \sin \delta-P_{2}^{+} \cos \left(\mu_{2}+\delta\right) \\
a_{44}=-\cos \left(\mu_{1}+\theta_{1}\right)
\end{gathered}
$$

and $A_{r}$ is the area ratio between $s^{+}$and $s_{\star}^{+}$. This matrix equation agrees with the results of Azevedo. 
The equations necessary to solve these individual terms are included in Sec. I.

There is, however, a subsystem of equations for $s_{\star}^{+}, \overline{E F}^{+}$, and $\overline{F H^{+}}$, which is sufficient to solve for the Mach stem height given that $s^{+}=A_{r} s_{\star}^{+}$.

$$
\left(\begin{array}{ccc}
A_{r} & a_{14} & a_{15} \\
a_{23} & a_{24} & a_{25} \\
1 & a_{54} & a_{55}
\end{array}\right)\left(\begin{array}{c}
s_{\star}^{+} \\
\frac{E F^{+}}{F H^{+}}
\end{array}\right)=\left(\begin{array}{c}
-\frac{1}{\gamma M_{\infty}^{2}} \cos \theta_{1}+P_{1}^{+} \cos \theta_{1} \\
0 \\
g^{+}
\end{array}\right)
$$

In this case the Mach stem height would be calculated using the area relationship of the sonic throat behind the Mach stem,

$$
s^{+}=A_{r} s_{\star}^{+}
$$

The analysis of Azevedo produces a geometry which is not selfconsistent. Specifically, the pressure in region 2 is taken to be constant, which is not consistent with the fact that the flow in region 3 is of varying pressure. Furthermore, there are two other equally valid solutions for $s^{+}$, besides that given in Eq. (15).

Specifically, as Azevedo writes,

$$
s^{+}=g^{+}+\sin \theta_{1}-\left(x_{s}^{+}+\cos \theta_{1}\right) \tan \alpha
$$

which states that the height of the Mach stem must be equal to $g$ plus the height of the wedge minus the height of the incident shock. Another equally valid way of writing the Mach stem height is

$$
s^{+}=s_{\star}^{+}+x_{\star}^{+} \tan \delta
$$

All three of these calculations, Eqs. (15-17), for the Mach stem height produce slightly different answers. Again, this is due to the fact that the pressure in region 3 varies and is not consistent with the assumption of constant pressure in region 2. It is important to note that there is no simple way of matching the pressure across the slip line. Because the pressure is not correct a solution that tries to conserve momentum is also incorrect and produces an inconsistent geometry. Therefore, it may be useful to fix the geometry and continue to allow the pressure across the slip line to be mismatched.

\section{Geometric Solution}

In Azevedo's solution the most restrictive assumption is that the sonic throat occurs at the leading characteristic of the expansion fan. Also, Azevedo does not force the geometry to be self-consistent, specifically, the condition that the slip line $\overline{T H}$, the expansion wave $\overline{F H}$, and the sonic throat intersect at a point is not imposed. To solve the latter problem we can write five equations that fix the geometry, assuming that all shocks and slip lines are straight. These equations are given in Sec. II and can be written in matrix form as

$$
\begin{aligned}
& \left(\begin{array}{ccccc}
\sin \alpha & A_{r} & 0 & 0 & 0 \\
0 & 1 & \sin \left(\delta+\mu_{2}\right) & \sin \left(\mu_{1}+\theta_{1}\right) & 0 \\
-\cos \alpha & -\cot \delta\left(A_{r}-1\right) & \cos \left(\delta+\mu_{2}\right) & \cos \left(\mu_{1}+\theta_{1}\right) & 0 \\
-\cos \alpha & 0 & 0 & \cos \left(\mu_{1}+\theta_{1}\right) & -\cos \phi \\
-\sin \alpha & 0 & 0 & \sin \left(\mu_{1}+\theta_{1}\right) & \sin \phi
\end{array}\right) \\
& \times\left(\begin{array}{c}
\overline{O T^{+}} \\
s_{\star}^{+} \\
\overline{F H^{+}} \\
\overline{E F^{+}} \\
\overline{T F^{+}}
\end{array}\right)=\left(\begin{array}{c}
g^{+}+\sin \theta_{1} \\
g^{+} \\
-\cos \theta_{1} \\
-\cos \theta_{1} \\
-\sin \theta_{1}
\end{array}\right)
\end{aligned}
$$

Finally, the Mach stem height $s$ can be calculated using Eq. (15). These equations, unlike those used by Azevedo, do not explicitly include the conservation of mass and momentum. However, if we consider the slip line to be a solid wall, we see that mass and momentum are conserved. This is because if the slip line were a solid wall the assumptions made in this analysis are exact, because the

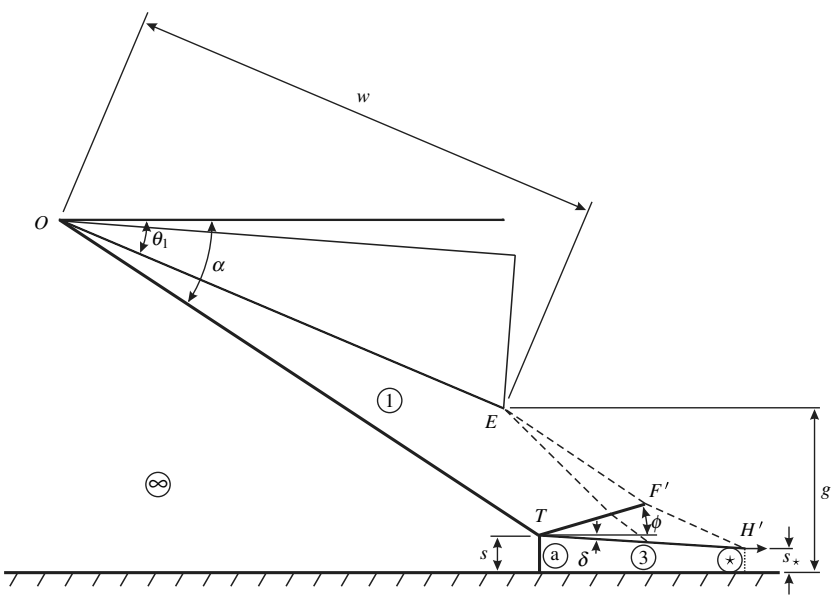

Fig. 2 Illustration of flow setup used to predict the Mach stem height.

entire geometry is self-consistent, and all of the shock jump conditions used conserve mass and momentum. Unfortunately, like Azevedo's solution, the pressure across the slip line is not continuous.

\section{Generalized Geometric Solution}

The problem still remains that all of these solutions assume that the sonic throat of the flow behind the Mach stem occurs at the leading characteristic of the expansion fan. To eliminate this problem, we will allow the sonic throat to occur further downstream. This generalized setup is shown in Fig. 2. The geometrical considerations are the same as those leading to Eq. (18), with $F$ and $H$ replaced by $F^{\prime}$ and $H^{\prime}$, respectively. Also, $\mu_{1^{\prime}}$ and $\overline{\mu_{2^{\prime}}}$ refer to the Mach angle along the characteristic corresponding to the sonic throat, rather than along the leading characteristic. A key point of this theory, like that of $\mathrm{Li}$ and Ben-Dor [4], is that the flow right above the sonic throat is parallel to the freestream flow.

$$
\begin{aligned}
& \left(\begin{array}{ccccc}
\sin \alpha & A_{r} & 0 & 0 & 0 \\
0 & 1 & \sin \left(\delta+\mu_{2^{\prime}}\right) & \sin \left(\mu_{1^{\prime}}+\theta_{1}\right) & 0 \\
-\cos \alpha & \cot \delta\left(1-A_{r}\right) & \cos \left(\delta+\mu_{2^{\prime}}\right) & \cos \left(\mu_{1^{\prime}}+\theta_{1}\right) & 0 \\
-\cos \alpha & 0 & 0 & \cos \left(\mu_{1^{\prime}}+\theta_{1}\right) & -\cos \phi \\
-\sin \alpha & 0 & 0 & \sin \left(\mu_{1^{\prime}}+\theta_{1}\right) & \sin \phi
\end{array}\right) \\
& \times\left(\begin{array}{c}
\overline{O T^{+}} \\
\overline{s_{\star}^{+}} \\
\overline{F^{\prime} H^{+}} \\
\overline{E F^{+}} \\
\overline{T F^{\prime}}
\end{array}\right)=\left(\begin{array}{c}
g^{+}+\sin \theta_{1} \\
g^{+} \\
-\cos \theta_{1} \\
-\cos \theta_{1} \\
-\sin \theta_{1}
\end{array}\right)
\end{aligned}
$$

$\delta$ and $\phi$ are calculated as before using triple-point theory. $\mu_{2^{\prime}}$ is calculated knowing that the flow just above the slip line must turn through an angle of $\delta$ to be parallel to the freestream just above the sonic throat,

$$
\delta=v\left(M_{2^{\prime}}\right)-v\left(M_{2}\right)
$$

where the Prandtl-Meyer function $v$ is defined as

$$
\nu(M)=\sqrt{\frac{\gamma+1}{\gamma-1}} \tan ^{-1} \sqrt{\frac{\gamma-1}{\gamma+1}\left(M^{2}-1\right)}-\tan ^{-1} \sqrt{M^{2}-1}
$$

Because $M_{2}$ is known from Eq. (A24), solutions for $M_{2^{\prime}}$ and $\mu_{2^{\prime}}$ may be obtained. $\mu_{1}$ is more difficult to determine because the flow deflection angle is not simply $\delta$, because the flow has passed through part of the expansion before it reaches the shock, as opposed to the flow in region 2, which first goes through the reflected shock. This 


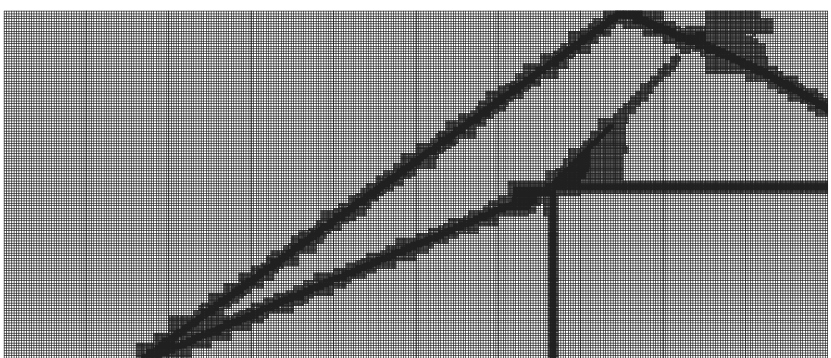

Fig. 3 Representative mesh refinement for the calculation of the Mach stem height using Amrita.

means that the flow in region 1 must turn through an angle of $\delta+\delta_{e}$, where $\delta_{e}$ is the extra turning required to compensate for the fact that the reflected shock is weaker at $F^{\prime}$ than at $T$. Specifically, the reflected shock at the point $F^{\prime}$ must turn the flow through $\theta_{1}-\delta-\delta_{e}$. Using oblique shock relations produces

$$
\begin{aligned}
& \cot \left(\theta_{1}-\delta-\delta_{e}\right)=\tan \left(\theta_{1}-\delta-\delta_{e}+\phi\right) \\
& \quad \times\left(\frac{(\gamma+1) M_{1^{\prime}}^{2}}{2\left(M_{1^{\prime}}^{2} \sin ^{2}\left(\theta_{1}-\delta-\delta_{\mathrm{e}}+\phi\right)-1\right)}-1\right)
\end{aligned}
$$

where $M_{1^{\prime}}$ is given by the Prandtl-Meyer function,

$$
\delta+\delta_{e}=v\left(M_{1^{\prime}}\right)-v\left(M_{1}\right)
$$

Equations (22) and (23) can then be solved simultaneously for $\delta_{e}$ and $M_{1^{\prime}}$. At this point $\mu_{1^{\prime}}$ is known and Eq. (19) can be solved. Once the matrix equation has been solved, the Mach stem height $s$ can be calculated using Eq. (15).

\section{Numerical Calculations}

Numerical simulations of the flow were performed for various conditions using the AMRITA software system. This software system has been constructed by Quirk [10]. It is a system that automates and packages computational tasks in such a way that the packages can be combined (dynamically linked) according to instructions written in a high-level scripting language. The present application uses features of AMRITA that include the automatic construction of an Euler solver, automatic adaptive mesh refinement according to simply chosen criteria, and scripting-language-driven computation and postprocessing of the results. The Euler solver generated for the present computation was an operator-split scheme with Harten-Lax-van Leer (HLLE) flux and kappa-monotone upstream-centered schemes for conservation laws (MUSCL) reconstruction.

The coarse grid for the Mach stem height calculations was $330 \times 140$, to which two levels of adaptive mesh refinement by a factor of 2 were applied. This results in an effective grid of $1320 \times 560$. The mesh was refined based on a density gradient criterion as well as along the surface of the wedge. An example of the grid is shown in Fig. $\underline{3}$, with an enlarged section shown in Fig. 4 .

\section{Mach Stem Height Results}

A comparison of the current theory with that of Azevedo [1,2] as well as the theory of $\mathrm{Li}$ and Ben-Dor [4], the numerical results of AMRITA, the experimental results of Hornung and Robinson [5], and the computations of Vuillon et al. [11] is shown in Fig. 5. This figure shows the significant improvement made using the generalized geometric solution. Overall agreement with the experimental data of Hornung and Robinson as well as with computational results is good. Of course differences between theory, computations, and experiments remain. There are several reasons for these discrepancy. First, the slip line originating from the triple point is not in fact straight. Second, the reflected shock will curve through the expansion fan. Third, viscous effects will cause a shear layer with negative displacement effect to develop along the slip line.

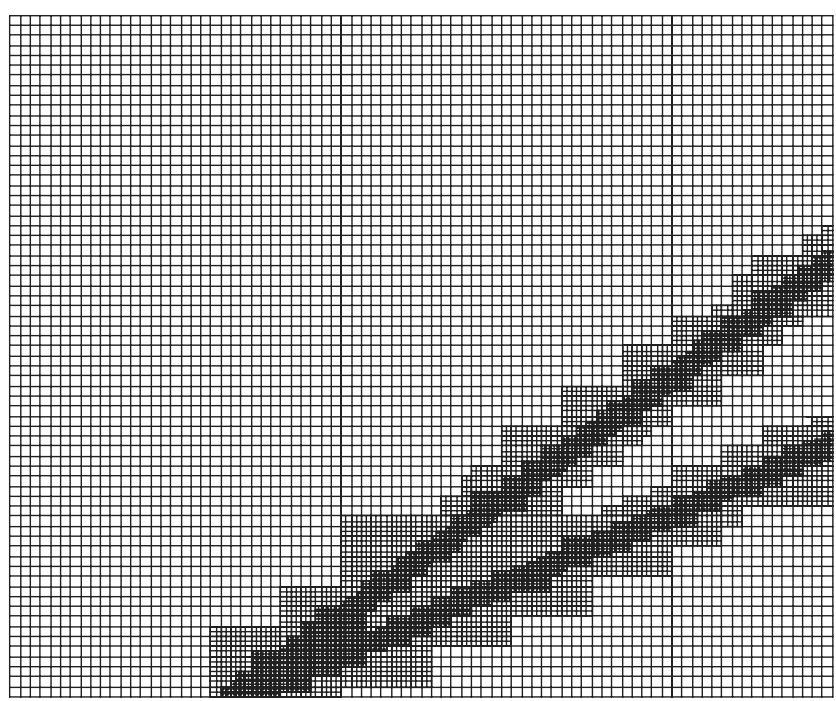

Fig. 4 Englarged region of a representative mesh refinement for the calculation of the Mach stem height using Amrita.

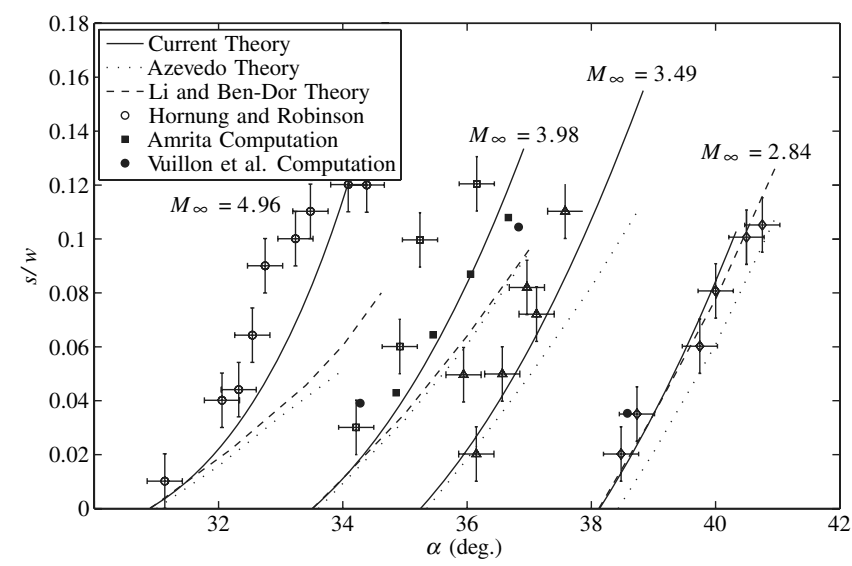

Fig. 5 Comparison of current Mach stem height calculations against those of Azevedo [1,2] and of $\mathrm{Li}$ [4], measurements by Hornung and Robinson [5], computations by Vuillon et al. [11], as well as current computations done using Amrita. $\gamma=1.4$ and $g / w \approx 0.4$.

It is important to note that the data which both Azevedo [1,2] and Li and Ben-Dor [4] attribute to Hornung and Robinson [5] are in fact not the data presented in that paper. The actual data of Hornung and Robinson are significantly different and show higher Mach stem heights than what is presented by Azevedo and by Li and Ben-Dor. This discrepancy is not obvious by simple visual inspection, because Hornung and Robinson normalize the Mach stem height by $w$, whereas others normalize the Mach stem height by $L$. Figure 5 shows the actual results presented by Hornung and Robinson. Azevedo and Liu [2] attribute this same data to a personal communication with Hornung. This personal communication consisted of Azevedo personally remeasuring the schlieren photographs of Hornung and Robinson [5]. As Hornung and Robinson note in their paper, many of their results suffered from wall interference effects, and as a result they did not use these data to determine the Mach stem height. Although no record exists of which schlieren photographs Azevedo made his measurements from, it is believed that most of the results he presents in his thesis [1], and which have been subsequently used by later authors, were from the schlieren images with wall interference effects.

\section{Moving Triple-Point Analysis}

The triple-point analysis presented earlier assumed a stationary Mach stem. We will now consider the case where the Mach stem 


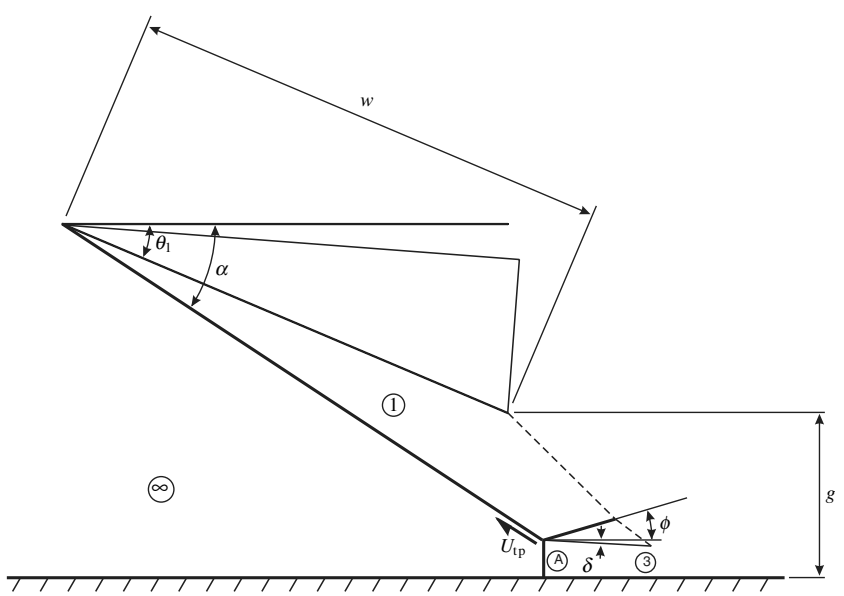

Fig. 6 Illustration of flow setup used for the Amrita simulations.

moves with an upstream velocity $U_{\mathrm{Ms}}$. This may occur in a steady freestream, for example, if transition to Mach reflection is initiated by some disturbance when the flow is initially in the dual-solution region. The rate at which the Mach stem moves upstream $U_{\mathrm{Ms}}$ is related to the speed at which the triple point travels up along the lead shock $U_{\text {tp }}$ by

$$
U_{\mathrm{tp}}=\frac{U_{\mathrm{Ms}}}{\cos \alpha}
$$

Figure 6 shows the flow setup when the triple point is moving.

To perform the triple-point analysis we must examine the flow both in the lab-fixed reference frame and in the frame of reference of the triple point. For this triple-point analysis we will consider a Mach stem that is not perpendicular to the flow, as is shown in Fig. 7. Quantities calculated in the reference frame of the triple point are denoted with a superscript ${ }^{\text {tp }}$. The flow Mach number coming into the Mach stem, $M_{\infty}^{\mathrm{tp}}$ is

$$
M_{\infty}^{\mathrm{tp}}=\sqrt{\left(M_{\infty}+M_{\mathrm{tp}} \cos \alpha\right)^{2}+\left(M_{\mathrm{tp}} \sin \alpha\right)^{2}}
$$

where

$$
M_{\mathrm{tp}}=\frac{U_{\mathrm{tp}}}{a_{\infty}}
$$

Because the leading oblique shock is stationary in the lab frame we can write the Mach number in region $1 M_{1}$ and the normalized pressure in region $1 P_{1}^{+}$as

$$
\begin{gathered}
P_{1}^{+}=\xi\left(M_{\infty}, \gamma, \alpha\right) \\
M_{1}=M\left(M_{\infty}, \gamma, \alpha\right)
\end{gathered}
$$

To calculate the flow in region 2 , we must consider the flow first in the reference frame of the triple point. The normalized pressure, Mach number, and flow angle in region 2 can be written as

$$
\begin{gathered}
P_{2}^{+}=P_{1}^{+} \xi\left(M_{1}^{\mathrm{tp}}, \gamma, \phi^{\mathrm{t}}\right) \\
M_{2}^{\mathrm{tp}}=M\left(M_{1}^{\mathrm{tp}}, \gamma, \phi^{\mathrm{tp}}\right) \\
\theta_{2}^{\mathrm{tp}}=\theta_{1}^{\mathrm{tp}}-\theta\left(M_{1}^{\mathrm{tp}}, \gamma, \phi^{\mathrm{tp}}\right)
\end{gathered}
$$

where

$M_{1}^{\mathrm{tp}}=\left(\frac{\left(M_{1} a_{1} \cos \theta_{1}+U_{\mathrm{tp}} \cos \alpha\right)^{2}+\left(M_{1} a_{1} \sin \theta_{1}+U_{\mathrm{tp}} \sin \alpha\right)^{2}}{a_{1}^{2}}\right)^{1 / 2}$

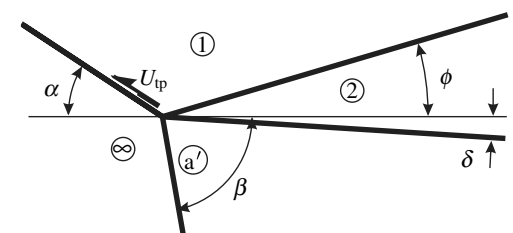

Fig. 7 Illustration of a moving triple point with a Mach stem that is not perpendicular to the flow.

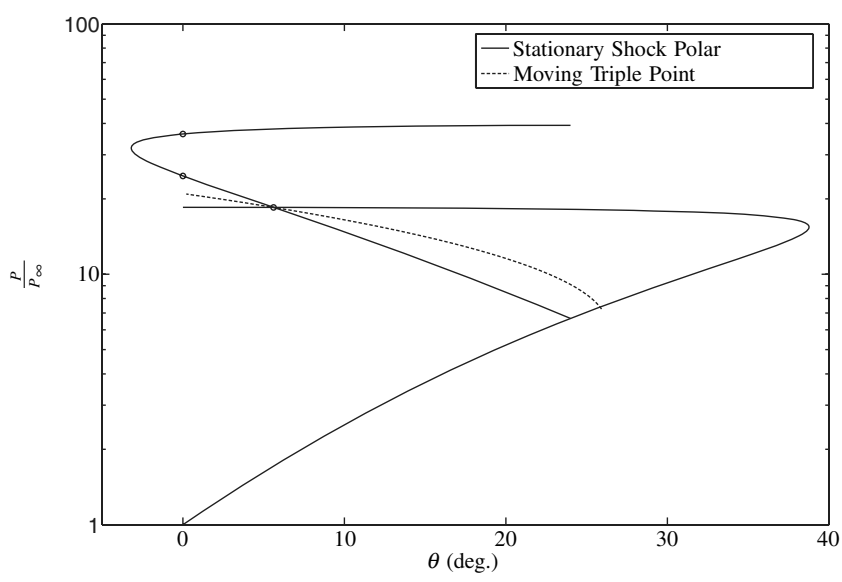

Fig. 8 Shock polar illustrating the effects of a moving Mach stem. In the case where the Mach stem is moving upstream the pressure ratio is higher than the stationary-case value, and vice versa. Each point on the moving triple-point curve represents the pressure and deflection angle for a given $M_{\mathrm{tp}} \cdot M_{\infty}=4, \gamma=1.4$, and $\theta_{w}=24 \mathrm{deg}$.

$$
\theta_{1}^{\mathrm{tp}}=\tan ^{-1} \frac{M_{1} a_{1} \sin \theta_{1}+U_{\mathrm{tp}} \sin \alpha}{M_{1} a_{1} \cos \theta_{1}+U_{\mathrm{tp}} \cos \alpha}
$$

Converting back into the lab-fixed coordinates, we see that

$$
\phi=\phi^{\mathrm{tp}}-\theta_{1}^{\mathrm{tp}}
$$

Similarly, the normalized pressure, Mach number, and flow angle in region $a^{\prime}$ can be written as

$$
\begin{gathered}
P_{a^{\prime}}^{+}=\xi\left(M_{\infty}^{\mathrm{tp}}, \gamma, \beta^{\mathrm{tp}}\right) \\
M_{a^{\prime}}^{\mathrm{tp}}=M\left(M_{\infty}^{\mathrm{tp}}, \gamma, \beta^{\mathrm{tp}}\right)
\end{gathered}
$$

$$
\theta_{a^{\prime}}^{\mathrm{tp}}=\theta_{\infty}^{\mathrm{tp}}-\theta\left(M_{\infty}^{\mathrm{tp}}, \gamma, \beta^{\mathrm{tp}}\right)
$$

where

$$
\begin{gathered}
M_{\infty}^{\mathrm{tp}}=\sqrt{\left(M_{\infty}+M_{\mathrm{tp}} \cos \alpha\right)^{2}+\left(M_{\mathrm{tp}} \sin \alpha\right)^{2}} \\
\theta_{\infty}^{\mathrm{tp}}=\tan ^{-1} \frac{M_{\mathrm{tp}} \sin \alpha}{M_{\infty}+M_{\mathrm{tp}} \cos \alpha}
\end{gathered}
$$

Converting these values back into lab-fixed coordinates produces

$$
\beta=\beta^{\mathrm{tp}}-\theta_{\infty}^{\mathrm{tp}}
$$

$$
\delta_{a^{\prime}}=\frac{M_{a^{\prime}}^{\mathrm{tp}} a_{a^{\prime}} \cos \theta_{a^{\prime}}^{\mathrm{tp}}-U_{\mathrm{tp}} \cos \alpha}{M_{a^{\prime}}^{\mathrm{tp}} a_{a^{\prime}} \sin \theta_{a^{\prime}}^{\mathrm{tp}}-U_{\mathrm{tp}} \sin \alpha}
$$

where $a_{a^{\prime}}$ is the speed of sound in region $a^{\prime}$. 
As we did earlier in Eqs. (르) and (A26), we impose

$$
\begin{gathered}
P_{2}^{+}=P_{a^{\prime}}^{+} \\
\delta_{2}=\delta_{a^{\prime}}
\end{gathered}
$$

The effect of a moving Mach stem is shown in Fig. 8. As one would expect, if the Mach stem is moving upstream, the pressure behind the Mach stem is higher, and if the Mach steam moves downstream the pressure is lower than in the stationary case. The speed at which the Mach stem can move downstream is limited by the fact that a reflected shock must be able to exist. Specifically, it is not possible for the triple point to be moving downstream so fast that the relative flow into the reflected shock is subsonic. This means that the perpendicular component of the flow into the reflected shock must be supersonic. As the flow speed into the reflected shock decreases, the pressure rise across the reflected shock also decreases, and we would

$$
\begin{gathered}
0 \\
\sin \left(\delta+\mu_{2}\left(U_{\mathrm{Ms}}\right)\right. \\
\cos \left(\delta+\mu_{2}\right)\left(U_{\mathrm{Ms}}\right) \\
0 \\
0
\end{gathered}
$$

stem in the lab frame was low and therefore there was a large stem-tothroat area ratio. Although it is required that the slip-line angle be less than in the steady-state case, it is not required that the Mach number behind the Mach stem be low, it is only helpful.

From Fig. 8, we see that the deflection angle is decreased if the shock is moving upstream. Additionally, the flow Mach number behind the Mach stem will decrease if the Mach stem moves upstream, which produces a large area ratio. Based on this we can hypothesize that for small Mach stems, the Mach stem must travel upstream. Based simply on geometry, a Mach stem traveling upstream also increases in height.

The moving Mach stem changes the slip-line angle $\delta$, the reflected shock angle $\phi$, the Mach angle in region $2^{\prime}$, and the area ratio between the Mach stem and the sonic throat $A_{r}$. Assuming quasi-steady flow, that is to say the speed at which the triple point grows is slow compared to the flow speed, Eq. (18), with modifications taking into account the moving Mach stem, becomes expect the pressure and the flow deflection to be similar to that of the leading shock alone. In other words, as the triple point moves downstream, the jump across the reflected shock becomes weaker and the flow deflection across the reflected shock decreases. This is indeed seen in Fig. $\underline{8}$, where the moving triple-point line terminates near the incident shock point.

\section{Mach Stem Height Variation}

As the Mach stem grows, it also slows down. Thus, for a given Mach stem speed a corresponding Mach stem height exists. Using Eq. (19) and substituting the modified flow parameters, as found in Sec. VIII, it is possible to calculate the Mach stem height at a given Mach stem speed. Conversely, given a Mach stem height, the Mach stem speed can be calculated. At the steady-state Mach stem height, the Mach stem velocity will of course be zero, because this is the definition of the steady-state height. Of special interest is the speed of the Mach stem when the height is different than the steady-state height, in particular, the speed of the Mach stem during the Mach stem growth phase.

To understand the growth phase of the Mach stem, let us consider a very small Mach stem, as shown in Fig. 9. If the Mach stem were stationary, the slip line originating from the triple point would have a finite angle and therefore reach the wall before the leading characteristic. Because it is not physically possible for the slip line to intersect the wall we know that this solution cannot be correct, and therefore the Mach stem must move in order to produce a different slip-line angle. Specifically, we need the slip-line angle to be at a small enough angle such that it reaches the first characteristic. We therefore now know that the triple point must move in a way as to decrease the slip-line angle. Let us now consider a slip-line angle sufficiently small that it intersects the first characteristic just above the wall. In this case, the area ratio between the Mach stem and the intersection of the slip line with the first characteristic would be very large. This means that, in addition to the slip line being sufficiently small, it would also be helpful if the Mach number behind the Mach where $\left(U_{\mathrm{Ms}}\right)$ denotes the value depending on the speed of the Mach stem. These modified values are derived from Eqs. (30), (34), (38), and (41).

Figure 10 illustrates the solution to the relationship between Mach stem velocity and Mach stem height as given by Eq. (44).

Given the numerical relationship between $M_{\mathrm{tp}}$ and $s / w$ it is possible to calculate the evolution of the Mach stem. Specifically,

$$
\frac{\mathrm{d} s}{\mathrm{~d} t}=U_{\mathrm{tp}} \sin \alpha
$$

In nondimensional form, this becomes

$$
\frac{d(s / w)}{d\left(t a_{\infty} / w\right)}=M_{\mathrm{tp}} \sin \alpha
$$

where $M_{\mathrm{tp}}$ is dependent on $s / w, M_{\infty}, g / w, \gamma$, and $\theta_{1}$. The calculation is quite straightforward and a comparison between the expected Mach stem growth and a numerical calculation done using AMRITA is shown in Fig. 11. Figure 5 shows that the steady-state Mach stem height grows rapidly with wedge angle. This means that even small errors can result in a large height difference. It is therefore reasonable to expect better agreement at lower steady-state Mach stem heights. Figure 12 shows the comparison of the Mach stem height growth for a wedge angle of $\theta_{1}=23 \mathrm{deg}$.

In Fig. 11 we see that the predicted Mach stem height is about $60 \%$ greater than in the numerical computation; however, the general growth trends show good agreement. We see in Fig. 13 that there is a significant difference between the shape of the slip line originating from the triple point and the slip line used in the theoretical estimate. Specifically, the computed slip line gradually approaches 0 deg thereby giving it a lower average angle. This lower average angle causes a decrease in the Mach stem height. For the cases considered, the theory appears to locate the throat formed by the flow downstream of the Mach stem quite accurately. 


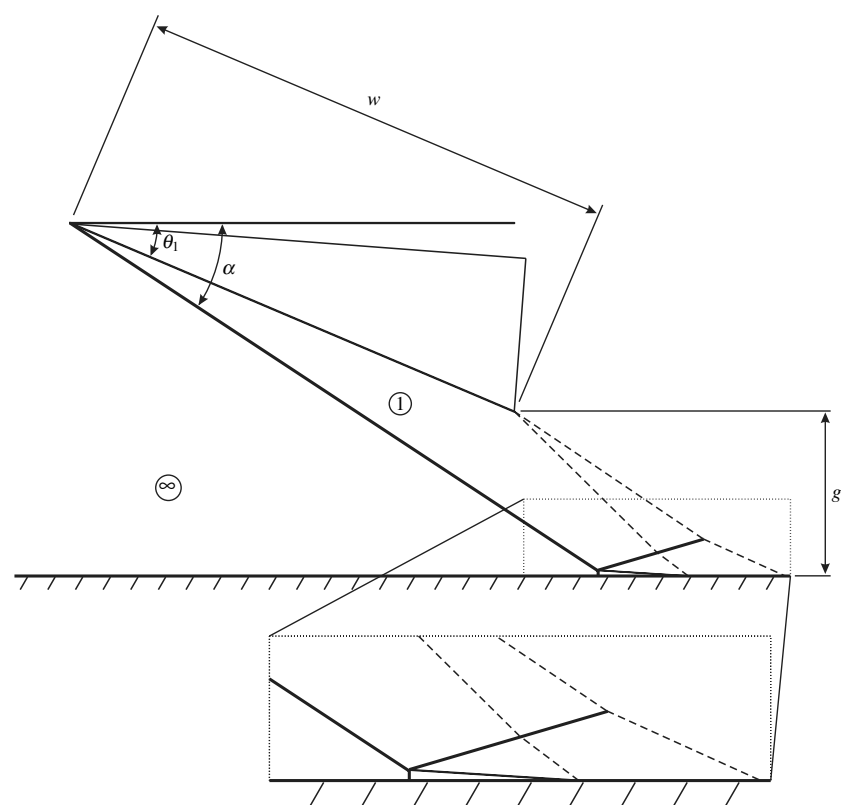

Fig. 9 Illustration of a very small Mach stem with a finite angle slip line. If the Mach stem is small enough, the slip line will intersect the axis of symmetry before it reaches the first expansion wave, which is not physically possible.

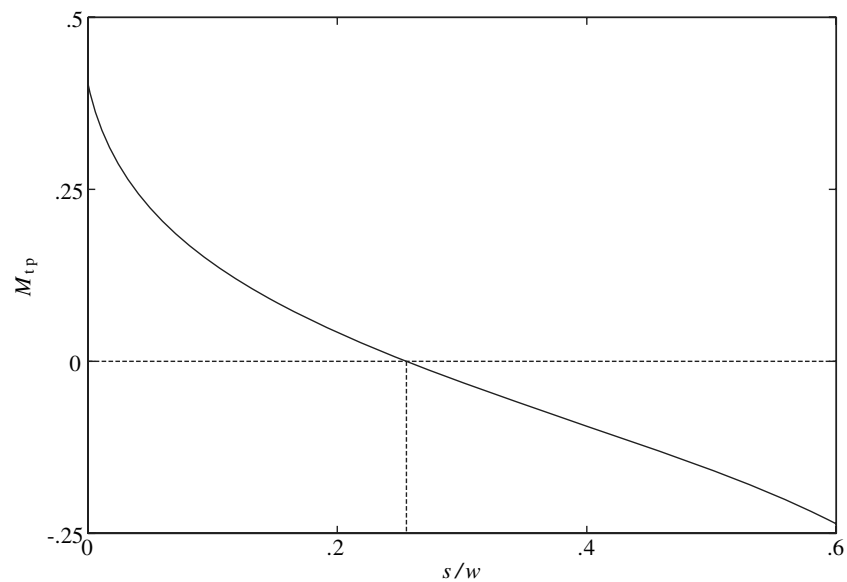

Fig. 10 Mach stem velocity as a function of Mach stem height based on Eq. (18). Positive $M_{\mathrm{tp}}$ indicates upstream speed. Calculated for $M_{\infty}=4$, $g / w=0.4, \gamma=1.4$, and $\theta_{1}=25 \mathrm{deg}$.

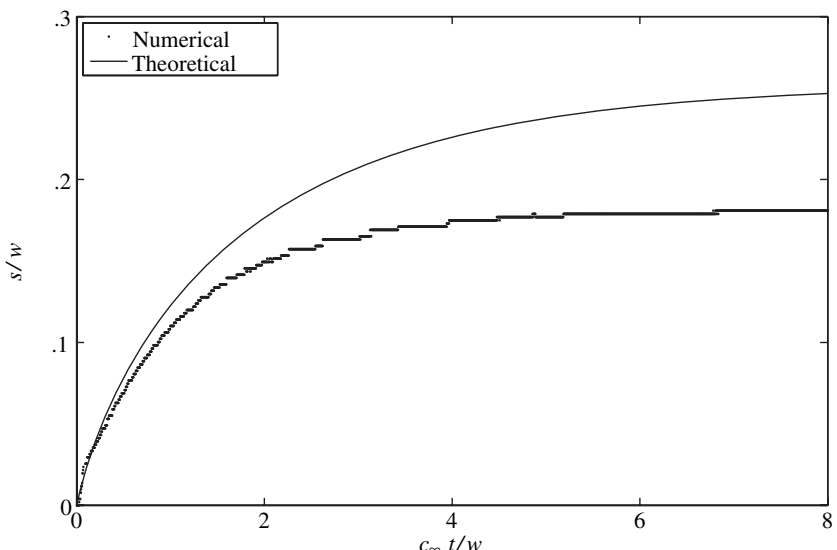

Fig. 11 Theoretical and numerical results for the height of the Mach stem as a function of time as it grows from an initial regular reflection condition. Calculated for $M_{\infty}=4, g / w=0.42, \quad \gamma=1.4$, and $\theta_{1}=25 \mathrm{deg}$.

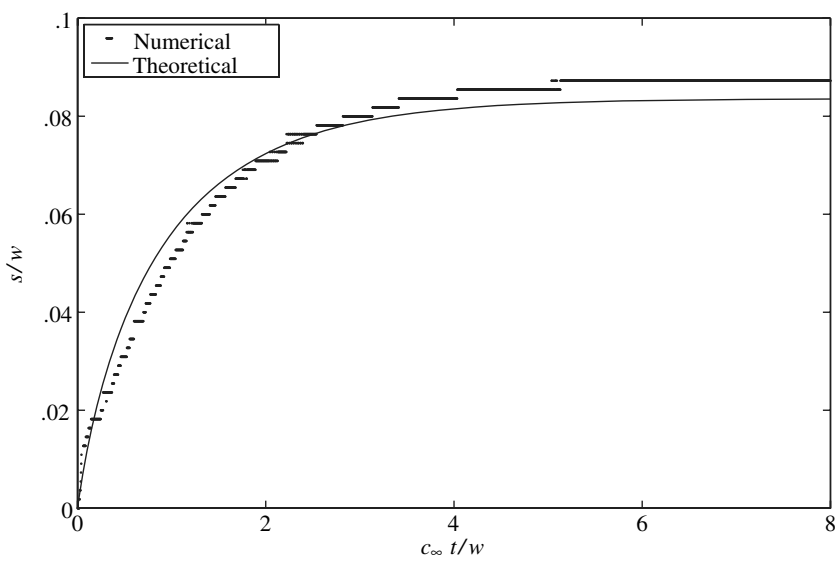

Fig. 12 Theoretical and numerical results for the height of the Mach stem as a function of time as it grows from an initial regular reflection condition. Calculated for $M_{\infty}=4, g / w=0.3907, \gamma=1.4$, and $\theta_{1}=23$ deg.

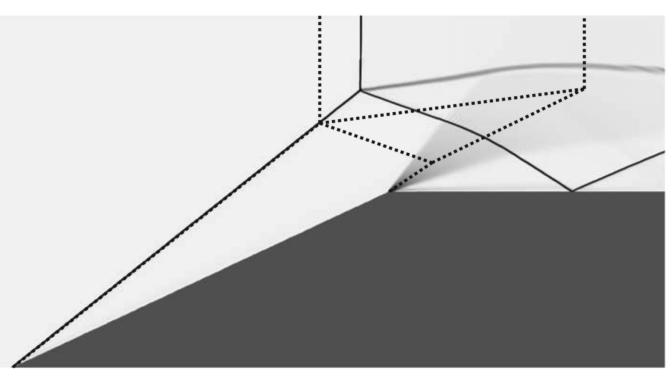

Fig. 13 A quasi-schlieren image showing a comparison between the theoretical shock structure and an Euler computation. The image shows that the shape of the slip line in the computation is significantly different than is assumed in the theory. This difference between computation and theory most likely accounts for the majority of the error between the two. The theoretical lines are shown as dotted lines. Calculated for $M_{\infty}=4$, $g / w=0.42, \gamma=1.4$, and $\theta_{1}=25 \mathrm{deg}$.

\section{Three-Dimensional Mach Stem Growth}

Consider a three-dimensional flow with a regular reflection in the dual-solution domain. When a Mach stem is first formed it is both small in height and in width in the spanwise direction. As it grows it both increases in height and expands outward in the spanwise direction. This opening is referred to as a mouth because of its shape [12]. The spanwise region where the transition from a Mach stem to a regular reflection occurs is characterized by a five-point theory. This point exists at the intersection of five shocks, those being the incoming shock, the regularly reflected shock, the Mach stem, the Mach stem reflected shock, and a fifth shock dividing the downstream flow region between the regular reflection and the Mach reflection. Further away from this point, we can expect the behavior of the Mach stem to follow that of the two-dimensional theory in the appropriate frame of reference. We can therefore conclude that the expansion rate of the Mach stem in the spanwise direction is determined by a complex system of five shocks, whereas the overall change in height of the Mach stem is governed by the twodimensional theory presented in Sec. V. Using the two-dimensional theory for the height and setting the spanwise expansion of the Mach stem to a constant, produces the evolution of a Mach stem that is seen in Fig. 14. This figure shows the Mach stem as it would be seen by an observer looking downstream. The assumption of a constant spanwise expansion is consistent with the dimensional analysis of the five-shock system and agrees very well with computational results.

This theory assumes that the Mach stem starts at a point and therefore predicts a cusp at the center of the Mach stem corresponding to the point where the Mach stem started. This, however, is not seen in computations and is believed to be due to the fact that in the computations the tripping from regular reflection to 


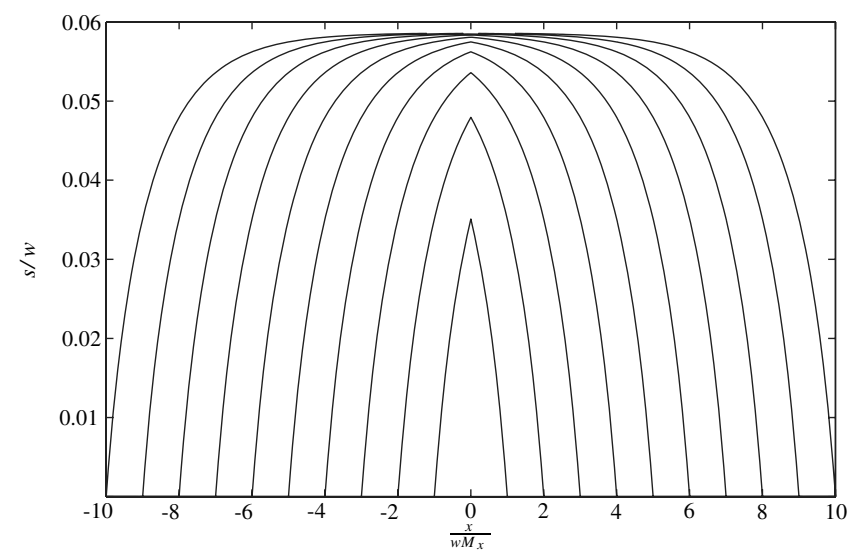

Fig. 14 Growth of Mach stem height $s$ and spanwise width of Mach $\operatorname{stem} x$, which is propagating outward at a Mach number $M_{x}$. Curves for flow times $\left(a_{\infty} t / w\right)$ between 1 and 10, in increments of 1 , with the lower curves corresponding to lower times. Calculated for $M_{\infty}=3$, $g / w=0.4516, w=150, \gamma=1.4$, and $\theta_{1}=21 \mathrm{deg}$.

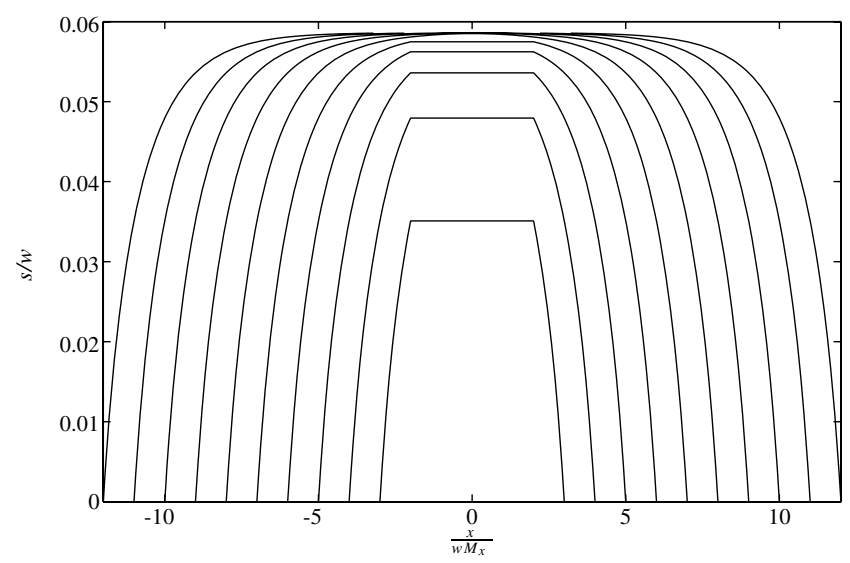

Fig. 15 Growth of Mach stem height considering a Mach stem with an initial finite width $s$ and spanwise width of Mach stem $x$, which is propagating outward at a Mach number $M_{x}$. Curves for flow times $\left(a_{\infty} t / w\right)$ between 1 and 10, in increments of 1 , with the lower curves corresponding to lower times. Calculated for $M_{\infty}=3, g / w=0.4516$, $w=150, \gamma=1.4$, and $\theta_{1}=21 \mathrm{deg}$.

Mach reflection occurs over a finite span portion of the regular reflection. This initial finite span is most likely due to the fact that the disturbance given is more than what is required to trip from regular reflection to Mach reflection. Essentially, in the computations the Mach stem starts with a finite width. For accurate comparisons with computations, an additional parameter, the initial width of the Mach stem, must be included. This initial width essentially separates the two halves of Fig. 14 and produces curves similar to Fig. 15 .

For comparison with three-dimensional calculations done using AMROC [13], the two-dimensional theory was used to calculate the change in height as a function of time at each spanwise point along the Mach stem. The expansion rate in the spanwise direction was taken to be a constant and was set to the best-fit value as was the initial Mach stem width. From dimensional analysis we can see that indeed the spanwise growth should be constant, because it will depend only on the local flow conditions around the five shock solutions; therefore,

$$
\frac{x}{c_{\infty} t}=h\left(M_{\infty}, \gamma, \theta_{w}\right)
$$

which gives a constant spanwise expansion speed for any given flow parameters. The use of the two-dimensional theory from Sec. VIII

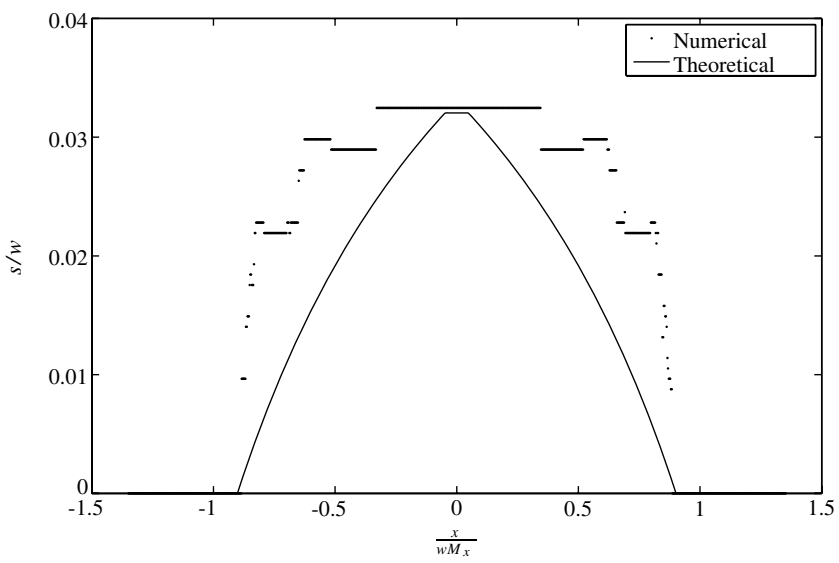

Fig. 16 Numerical and theoretical growth of Mach stem height $s$ and growth in the spanwise direction $x$ at $a_{\infty} t / w=0.11$. The Mach stem is propagating outward at a Mach number $M_{x}=0.5916$. Calculated for $M_{\infty}=3, g / w=0.4516, w=150, \gamma=1.4$, and $\theta_{1}=21 \mathrm{deg}$.

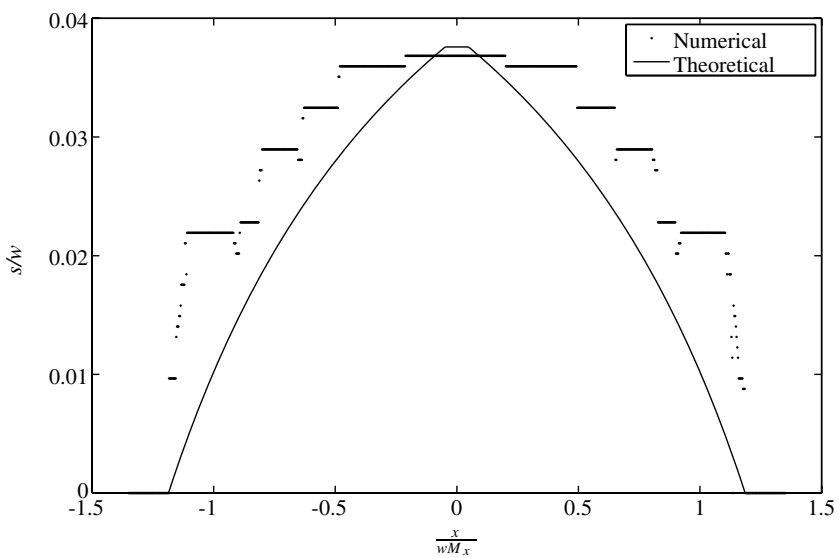

Fig. 17 Numerical and theoretical growth of Mach stem height $s$ and growth in the spanwise direction $x$ at $a_{\infty} t / w=0.39$. The Mach stem is propagating outward at a Mach number $M_{x}=0.5916$. Calculated for $M_{\infty}=3, g / w=0.4516, w=150, \gamma=1.4$, and $\theta_{1}=21 \mathrm{deg}$.

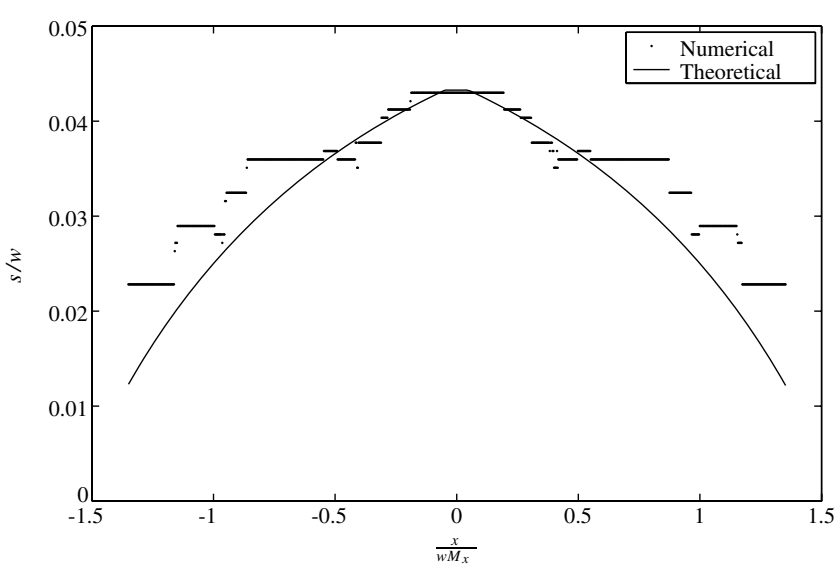

Fig. 18 Numerical and theoretical growth of Mach stem height $s$ and growth in the spanwise direction $x$ at $a_{\infty} t / w=0.79$. The Mach stem is propagating outward at a Mach number $M_{x}=0.5916$. Calculated for $M_{\infty}=3, g / w=0.4516, w=150, \gamma=1.4$, and $\theta_{1}=21 \mathrm{deg}$.

and the best-fit spanwise growth rate yields very good agreement to computations. Figures 16-18 show the progression of the threedimensional Mach stem with time, both computationally and theoretically. 


\section{Conclusions}

An approximate theory is presented for the Mach stem height and growth rates in both two and three dimensions. The calculation of Mach stem height shows a significant improvement over previous estimates and good agreement with both experiment and computations. A weakness in the theory lies in the assumptions relating to the slip line originating from the triple point. The theory could be improved by permitting the slip line to be curved, as Li and Ben-Dor [4] did, which would more accurately capture the true physics. However, such an improvement would drastically complicate the theory and produce a set of equations which would be significantly harder to solve.

The approximate theory predicts Mach stem growth rates that show good agreement in terms of both the time to reach the steadystate height as well as initial growth rate, while it does not do so well with the asymptotic Mach stem height. The fact that the growth rate is positive for small Mach stems is significant, because this tells us that, absent other disturbances, even the smallest Mach stem will grow until it reaches its steady-state height. It also shows that, after a small disturbance to the Mach stem, the Mach stem will return to its steadystate height. This indicates that a substantial disturbance would be required to cause transition from Mach reflection to regular reflection, because a disturbance that only shrinks and does not entirely remove the Mach stem will be insufficient. Computations by Kudryavtsev et al. [14] indicated that a relatively small disturbance would be able to cause transition from Mach reflection to regular reflection. Later, more refined computations by Khotyanovsky et al. [15] show that small disturbances are not sufficient to cause transition from Mach reflection to regular reflection, but can only temporarily decrease the height of the Mach stem height, which is consistent with the theory presented.

Good agreement between three-dimensional computations and the three-dimensional theory was observed. A weakness in the theory is the lack of an analytic approximation for the spanwise growth rate of the Mach stem. In addition, there are significant computational issues in accurately measuring the size of the Mach stem as a function of time. As is the case with the two-dimensional growth rate, a better estimate of the steady-state Mach stem height would improve the three-dimensional growth rate predictions.

\section{Appendix A: Mass and Momentum Balance}

Azevedo first considers the conservation of mass. Specifically, he considers the mass flow entering between the wedge tip $O$ and the symmetry plane. This mass flow can then be equated to the mass flow through $\overline{E F}, \overline{F H}$, and $s_{\star}$. Equating these two mass fluxes produces the following equation:

$$
\begin{aligned}
g^{+} & +\sin \theta_{1} \\
& =\rho_{1}^{+} u_{1}^{+} \sin \mu_{1} \overline{E F}^{+}+\rho_{2}^{+} u_{2}^{+} \sin \mu_{2} \overline{F H}^{+}+\rho_{\star}^{+} u_{\star}^{+} s_{\star}^{+}
\end{aligned}
$$

where $\mu_{1}$ and $\mu_{2}$ are the Mach angles and are given by

$$
\begin{aligned}
& \mu_{1}=\sin ^{-1} \frac{1}{M_{1}} \\
& \mu_{2}=\sin ^{-1} \frac{1}{M_{2}} .
\end{aligned}
$$

Next, he considers the conservation of momentum in the freestream flow direction. Equating the pressure and momentum flux between the wedge tip and the solid surface with the pressure and the momentum flux through $\overline{E F}, \overline{F H}$, and $s_{\star}$ produces

$$
\begin{aligned}
& P_{\infty}^{+}\left(g^{+}+\sin \theta_{1}\right)-P_{1}^{+}\left(\sin \theta_{1}+\sin \left(\mu_{1}+\theta_{1}\right) \overline{E F}^{+}\right) \\
& \quad-P_{2}^{+} \sin \left(\mu_{2}+\delta\right) \overline{F H^{+}}-P_{\star}^{+} s_{\star}^{+}=\rho_{1}^{+}\left(u_{1}^{+}\right)^{2} \sin \mu_{1} \cos \theta_{1} \overline{E F^{+}} \\
& \quad+\rho_{2}^{+}\left(u_{2}^{+}\right)^{2} \sin \mu_{2} \cos \delta \overline{F H}^{+}+\rho_{\star}^{+}\left(u_{\star}^{+}\right)^{2} s_{\star}^{+}-g^{+}-\sin \theta_{1}(\mathrm{~A} 4)
\end{aligned}
$$

Similarly, for conservation of momentum perpendicular to the freestream flow direction, he finds that

$$
\begin{aligned}
& P_{\infty}^{+}\left(x_{s}^{+}+\cos \theta_{1}\right)+P_{3}^{+} x_{\star}^{+}-P_{1}^{+}\left(\cos \theta_{1}+\cos \left(\mu_{1}+\theta_{1}\right) \overline{E F}^{+}\right) \\
& \quad-P_{2}^{+} \cos \left(\mu_{2}+\delta\right) \overline{F H^{+}}=-\rho_{1}^{+}\left(u_{1}^{+}\right)^{2} \sin \mu_{1} \sin \theta_{1} \overline{E F}^{+} \\
& \quad-\rho_{2}^{+}\left(u_{2}^{+}\right)^{2} \sin \mu_{2} \sin \delta \overline{F H^{+}}
\end{aligned}
$$

Azevedo takes $P_{3}$ to be the average pressure in region 3, which is the average of the pressure at the sonic throat and the pressure right behind the Mach stem. The numerical result is almost identical if we take $P_{3}$ to be the integrated pressure using the area ratio relationship.

The superscript + refers to nondimensional quantities. Specifically, density is normalized by the freestream density $\rho_{\infty}$, velocities are normalized by the freestream velocity $u_{\infty}$, pressures are normalized by twice the freestream dynamic pressure $\rho_{\infty} u_{\infty}^{2}$, and distances are normalized by the wedge length $w$.

We can now apply the shock jump conditions and the equation of state for a perfect gas. Specifically, we will use the following relations:

$$
\begin{gathered}
\rho_{1}^{+} u_{1}^{+}=\frac{\sin \alpha}{\sin \left(\alpha-\theta_{1}\right)} \\
\rho_{2}^{+} u_{2}^{+}=\frac{\sin \alpha \sin \left(\theta_{1}+\phi\right)}{\sin \left(\alpha-\theta_{1}\right) \sin (\phi+\delta)} \\
\rho_{\star}^{+} u_{\star}^{+}=\frac{s^{+}}{s_{\star}^{+}} \\
\rho_{1}^{+}\left(u_{1}^{+}\right)^{2}=\gamma P_{1}^{+} M_{1}^{2} \\
\rho_{2}^{+}\left(u_{2}^{+}\right)^{2}=\gamma P_{2}^{+} M_{2}^{2} \\
\rho_{\star}^{+}\left(u_{\star}^{+}\right)^{2}=\gamma P_{\star}^{+}
\end{gathered}
$$

With these relations we can rewrite Eqs. (1ㅡ는 $)$ as

$$
\begin{aligned}
g^{+} & +\sin \theta_{1}=\frac{\sin \alpha}{\sin \left(\alpha-\theta_{1}\right)} \sin \mu_{1} \overline{E F}^{+} \\
& +\frac{\sin \alpha \sin \left(\theta_{1}+\phi\right)}{\sin \left(\alpha-\theta_{1}\right) \sin (\phi+\delta)} \sin \mu_{2} \overline{F H}^{+}+s^{+}
\end{aligned}
$$

$$
\begin{aligned}
& \frac{1}{\gamma M_{\infty}^{2}}\left(g^{+}+\sin \theta_{1}\right)-P_{1}^{+}\left(\sin \theta_{1}+\sin \left(\mu_{1}+\theta_{1}\right) \overline{E F^{+}}\right) \\
& -P_{2}^{+} \sin \left(\mu_{2}+\delta\right) \overline{F H^{+}}-P_{\star}^{+} s_{\star}^{+}=\gamma P_{1}^{+} M_{1}^{2} \sin \mu_{1} \cos \theta_{1} \overline{E F}^{+} \\
& +\gamma P_{2}^{+} M_{2}^{2} \sin \mu_{2} \cos \delta \overline{F H^{+}}+\gamma P_{\star}^{+} s_{\star}^{+}-g^{+}-\sin \theta_{1}
\end{aligned}
$$

$$
\begin{aligned}
& \frac{1}{\gamma M_{\infty}^{2}}\left(x_{s}^{+}+\cos \theta_{1}\right)+P_{3}^{+} x_{\star}^{+}-P_{1}^{+}\left(\cos \theta_{1}+\cos \left(\mu_{1}+\theta_{1}\right) \overline{E F^{+}}\right) \\
& \quad-P_{2}^{+} \cos \left(\mu_{2}+\delta\right) \overline{F H^{+}}=-\gamma P_{1}^{+} M_{1}^{2} \sin \mu_{1} \sin \theta_{1} \overline{E F}^{+} \\
& -\gamma P_{2}^{+} M_{2}^{2} \sin \mu_{2} \sin \delta \overline{F H}^{+}
\end{aligned}
$$

At this point there are five unknowns, $x_{s}^{+}, x_{\star}^{+}, s_{\star}^{+}, \overline{E F}^{+}$, and $\overline{F H}^{+}$, but only three equations. Therefore, to close the system, Azevedo uses two geometric relationships:

$$
x_{\star}^{+}=\cos \left(\mu_{1}+\theta_{1}\right) \overline{E F}^{+}+\cos \left(\mu_{2}+\delta\right) \overline{F H}^{+}-x_{s}^{+}
$$




$$
s_{\star}^{+}=g^{+}-\sin \left(\mu_{1}+\theta_{1}\right) \overline{E F}^{+}-\sin \left(\mu_{2}+\delta\right) \overline{F H}^{+}
$$

Given the geometry, the Mach number, and the ratio of specific heats, all of the parameters of the matrix equation can be calculated. The shock angle $\alpha$, the Mach number, and the pressure behind the leading oblique shock, $M_{1}$ and $P_{1}$, can be calculated using the oblique shock relations:

$$
\begin{array}{r}
\cot \theta_{1}=\tan \alpha\left(\frac{(\gamma+1) M_{\infty}^{2}}{2\left(M_{\infty}^{2} \sin ^{2} \alpha-1\right)}-1\right) \\
P_{1}^{+}=\frac{1}{\gamma M_{\infty}^{2}} \frac{2 \gamma M_{\infty}^{2} \sin ^{2} \alpha-(\gamma-1)}{\gamma+1} \\
M_{1}^{2}=\frac{(\gamma-1) M_{\infty}^{2} \sin ^{2} \alpha+2}{\sin ^{2}\left(\alpha-\theta_{1}\right)\left(2 \gamma M_{\infty}^{2} \sin ^{2} \alpha-(\gamma-1)\right)}
\end{array}
$$

Because Azevedo assumes that the Mach stem is a normal shock, the Mach number and pressure just behind the Mach stem $M_{a}$ and $P_{a}$ can be found using the normal shock relations,

$$
\begin{gathered}
P_{a}^{+}=\frac{1}{\gamma M_{\infty}^{2}} \frac{2 \gamma M_{\infty}^{2}-(\gamma-1)}{\gamma+1} \\
M_{a}^{2}=\frac{(\gamma-1) M_{\infty}^{2}+2}{2 \gamma M_{\infty}^{2}-(\gamma-1)}
\end{gathered}
$$

Again using the oblique shock relations we find the flow angle, the pressure, and the Mach number in region 2 to be

$$
\begin{gathered}
\cot \left(\theta_{1}-\delta\right)=\tan \left(\phi+\theta_{1}\right)\left(\frac{(\gamma+1) M_{1}^{2}}{2\left(M_{1}^{2} \sin ^{2}\left(\phi+\theta_{1}\right)-1\right)}-1\right) \\
P_{2}^{+}=P_{1}^{+} \frac{2 \gamma M_{1}^{2} \sin ^{2}\left(\phi+\theta_{1}\right)-(\gamma-1)}{\gamma+1} \\
M_{2}^{2}=\frac{(\gamma-1) M_{1}^{2} \sin ^{2}\left(\phi+\theta_{1}\right)+2}{\sin ^{2}(\phi+\delta)\left(2 \gamma M_{\infty}^{2} \sin ^{2} \alpha-(\gamma-1)\right)}
\end{gathered}
$$

The angle of the slip line $\delta$ can be calculated using the triple-point theory. This theory states that the pressure and flow angle must be continuous across the slip line. Up to this point we have been assuming that the Mach stem is a normal shock; however, to accurately analyze the triple point, the angle of the Mach stem is important. A close-up of the triple point is shown in Fig. 7, for the steady-state case, $U_{/ \text {rmtp }}$ is taken to be zero. Note that the region behind the Mach stem is referred to as $a^{\prime}$ rather than $a$, for the purposes of calculating the triple-point deflection angle when an oblique Mach stem is used; whereas, for calculating the flow in the converging flow behind the Mach stem a normal Mach stem is considered. There is no closed form solution to this problem, so an iterative scheme must be used. The two following equations are solved for $\phi$ and $\beta$ :

$$
\begin{gathered}
P_{2}^{+}=P_{a^{\prime}}^{+} \\
\delta_{2}=\delta_{a^{\prime}}
\end{gathered}
$$

These equations are

$$
P_{1}^{+} \frac{2 \gamma M_{1}^{2} \sin ^{2}\left(\phi+\theta_{1}\right)-(\gamma-1)}{\gamma+1}=\frac{1}{\gamma M_{\infty}^{2}} \frac{2 \gamma M_{\infty}^{2} \sin ^{2} \beta-(\gamma-1)}{\gamma+1}
$$

$$
\begin{aligned}
& \tan \left(\phi+\theta_{1}\right)\left(\frac{(\gamma+1) M_{1}^{2}}{M_{1}^{2} \sin ^{2}\left(\phi+\theta_{1}\right)-1}-2\right) \\
& \quad=\tan \beta\left(\frac{(\gamma+1) M_{\infty}^{2}}{M_{\infty}^{2} \sin ^{2} \beta-1}-2\right)
\end{aligned}
$$

With $\phi$ known, Eq. (A22) can be used to solve for $\delta$. Finally, to solve Eq. (2), the area ratio $A_{r}$ and pressure ratio $P_{\star} / P_{a}$ of the converging flow behind the Mach stem must be calculated. These are

$$
\begin{gathered}
A_{r}=\frac{1}{M_{a}}\left(\frac{2}{\gamma+1}\left(1+\frac{\gamma-1}{2} M_{a}^{2}\right)\right)^{\frac{\gamma+1}{(\gamma-1)}} \\
\frac{P_{\star}}{P_{a}}=\left(\frac{2}{\gamma+1}\left(1+\frac{\gamma-1}{2} M_{a}^{2}\right)\right)^{\frac{\gamma}{\gamma-1}}
\end{gathered}
$$

since the point $\star$ corresponds to the sonic throat. At this point, all the terms in the matrix in Eq. (2) are known and the linear system can be solved. Finally, Azevedo solves for the Mach stem height by using the geometric relationship

$$
s^{+}=g^{+}+\sin \theta_{1}-\left(x_{s}^{+}+\cos \theta_{1}\right) \tan \alpha
$$

This equation illustrates that the problem is overdefined, because the above equation is not consistent with the area ratio relationship presented earlier.

\section{Appendix B: Geometric Solution}

$$
\begin{gathered}
\sin \alpha \overline{O T}^{+}+A_{r} s_{\star}^{+}=g^{+}+\sin \theta_{1} \\
s_{\star}^{+}+\sin \left(\delta+\mu_{2}\right) \overline{F H^{+}}+\sin \left(\mu_{1}+\theta_{1}\right) \overline{E F}^{+}=g^{+} \\
\cos \alpha \overline{O T^{+}}+\cot \delta\left(A_{r}-1\right) s_{\star}^{+}-\cos \left(\delta+\mu_{2}\right) \overline{F H^{+}} \\
-\cos \left(\mu_{1}+\theta_{1}\right) \overline{E F}^{+}=\cos \theta_{1} \\
\cos \alpha \overline{O T}^{+}+\cos \phi \overline{T F^{+}}-\cos \left(\mu_{1}+\theta_{1}\right) \overline{E F^{+}}=\cos \theta_{1} \\
\sin \alpha \overline{O T}^{+}-\sin \phi \overline{T F}^{+}-\sin \left(\mu_{1}+\theta_{1}\right) \overline{E F}^{+}=\sin \theta_{1}
\end{gathered}
$$

\section{Acknowledgments}

This work was funded by the U.S. Air Force Office of Scientific Research, under the supervision of John Schmisseur. The authors would also like to thank James Quirk and Ralf Deiterding for their assistance with AMRITA and AMROC, respectively, as well as Nicolas Ponchaut.

\section{References}

[1] Azevedo, D. J., "Analytic Prediction of Shock Patterns in a High-Speed, Wedge-Bounded Duct," Ph.D. Thesis, State University of New York, Buffalo, NY, 1989.

[2] Azevedo, D. J., and Liu, C. S., "Engineering Approach to the Prediction of Shock Patterns in Bounded High-Speed Flows," AIAA Journal, Vol. 31, No. 1, 1993, pp. 83-90.

[3] Ben-Dor, G., Shock Wave Reflection Phenomena, Springer-Verlag, New York, 1992. 
[4] Li, H., and Ben-Dor, G., "A Parametric Study of Mach Reflection in Steady Flows," Journal of Fluid Mechanics, Vol. 341, No. 1, 1997, pp. 101-125.

[5] Hornung, H. G., and Robinson, M. L., "Transition from Regular to Mach Reflection of Shock-Waves Part 2. The Steady-Flow Criterion," Journal of Fluid Mechanics, Vol. 123, 1982, pp. 155-164.

[6] Li, H., Schotz, M., and Ben-Dor, G., "Wave Configuration of Mach Reflection in Steady Flows: Analytical Solution and Dependence on Downstream Influences," International Symposium on Shock Waves, edited by B. Sturtevant, J. E. Shepherd, and H. G. Hornung, Vol. 1, Springer, Berlin, 1995, pp. 393-398.

[7] Schotz, M., Levy, A., Ben-Dor, G., and Igra, O., "Analytical Prediction of the Wave Configuration Size in Steady Flow Mach Reflections," Shock Waves, Vol. 7, No. 6, 1997, pp. 363-372.

[8] Ben-Dor, G., Elperin, T., Li, H., Vasiliev, E., Chpoun, A., and Zeitoun, D., "Dependence of Steady Mach Reflections on the Reflecting-Wedge Trailing-Edge Angle," AIAA Journal, Vol. 35, No. 11, 1997, pp. 17801782.

[9] Chpoun, A., and Leclerc, E., "Experimental Investigation of the Influence of Downstream Flow Conditions on Mach Stem Height," Shock Waves, Vol. 9, No. 4, 1999, pp. 269-271.

[10] Quirk, J. J., "Amrita—A Computational Facility (for CFD Modelling), "
VKI 29th CFD Lecture Series, Von Karman Institute for Fluid Dynamics, 1998.

[11] Vuillon, J., Zeitoun, D., and Ben-Dor, G., "Reconsideration of Oblique Shock Wave Reflection in Steady Flows. Part 2. Numerical Investigation," Journal of Fluid Mechanics, Vol. 301, 1995, pp. 37-50.

[12] Sudani, N., Sato, M., Karasawa, T., Noda, J., Tate, A., and Watanabe, M., "Irregular Effects on the Transition from Regular to Mach Reflection of Shock Wave in Wind Tunnel Flows," Journal of Fluid Mechanics, Vol. 459, 2002, pp. 167-185.

[13] Deiterding, R., "AMROC-Blockstructured Adaptive Mesh Refinement in Objectoriented C++," 2006, http://amroc.sourceforge.net/ [retrieved 6 June 2006]

[14] Kudryavtsev, A. N., Khotyanovsky, D. V., Ivanov, M. S., Hadjadj, A., and Vandromme, D., "Numerical Investigations of Transition Between Regular and Mach Reflections Caused by Free-Stream Disturbances," Shock Waves, Vol. 12, No. 2, 2002, pp. 157-165.

[15] Khotyanovsky, D. V., Kudryavtsev, A., and Ivanov, M. S., "Effects of a Single-Pulse Energy Deposition on Steady Shock Wave Reflection," Shock Waves, Vol. 15, No. 5, 2006, pp. 353-362.

A. Tumin Associate Editor 